\title{
Characteristic Fragmentation of Polysiloxane Monolayer Films by Bombardment with Monatomic and Polyatomic Primary lons in TOF-SIMS
}

\author{
Hye Kyoung Moon, David D. Wells, Joseph A. Gardella Jr.
}

Department of Chemistry, State University of New York at Buffalo, Buffalo, NY, 14260-3000, USA

\begin{abstract}
This study reports the characteristic fragmentation patterns from two polysiloxane polymers that form ordered overlayer on silver substrates. Results are compared for the bombardment of various monatomic and polyatomic projectiles of $\mathrm{Cs}^{+}, \mathrm{C}_{60}{ }^{+}(10 \mathrm{keV}), \mathrm{Bi}_{1}{ }^{+}$, and $\mathrm{Bi}_{3}{ }^{+}(25 \mathrm{keV})$ in the high mass range time-of-flight secondary ion mass spectrometry (TOF-SIMS) spectra. Results are reported from sub-monolayer (solution cast) coverages of poly(dimethylsiloxane)s with the number average molecular weights $\left(M_{n}\right)$ of 2200 and $6140 \mathrm{Da}$, respectively, and Langmuir-Blodgett monolayers of poly(methylphenylsiloxane) with molecular weights (MW) from 600 and $1000 \mathrm{Da}$. For each film, Bi projectiles resulted in the emission of positive silver cluster ions from the substrate under the polymer overlayer and peaks corresponding to silver cluster ions with larger mass were observed by impact of polyatomic $25 \mathrm{keV} \mathrm{Bi}_{3}{ }^{+}$projectiles. In addition, depending on the change of energy of $\mathrm{Bi}_{3}{ }^{+}$, a different pattern of fragments was observed. With $\mathrm{Cs}^{+}$and $\mathrm{C}_{60}{ }^{+}$impact, however, the emission of silver cluster ions was not detected. In the case of $\mathrm{C}_{60}{ }^{+}$impact for PDMS-6140, peaks corresponding to silver-cationized intact oligomers were not observed. In this paper, these results are explained by the possible bombardment mechanism for each projectile, based on its mass, energy, and split trajectories of the component atoms under the polyatomic impact.
\end{abstract}

Key words: TOF-SIMS, Polymer fragmentation, Cluster primary ion sources, Silicone polymers

\section{Introduction}

$\mathrm{T}$ he use of polyatomic ion sources in time-of-flight secondary ion mass spectrometry (TOF-SIMS) has recently been developed, promising the enhancement of molecular ion yields and therefore an increase in instrument sensitivity [1-3]. Compared with monatomic projectiles of comparable mass and energy, polyatomic projectiles lead to significant enhancement in the secondary ion formation efficiencies as well as increased secondary ion yield [1-9]. The magnitude of this yield enhancement by polyatomic projectiles is strongly influenced by the mass, energy, and the number of component atoms in the clusters [3]. The

Correspondence to: Joseph A. Gardella Jr.; e-mail: gardella@buffalo.edu mechanism of ion formation under cluster ion bombardment has yet to be completely explained. Theoretical and experimental studies, however, have shown the increased yield results from an overlap of low energy impact cascades of individual atomic components of cluster ions [10-14].

Various model systems constructed from overlayers of inorganic and organic films on surfaces have also been studied to determine the degree of desorption of molecular ions [3]. For example, under impact of polyatomic primary ions, the increase in secondary ion yield for organic multilayers on silver substrates was more pronounced than that for monolayer coverage $[15,16]$.

These previous experimental studies for particular projectile-sample combinations have mainly focused on the secondary ion yield, disappearance cross-section, and ion 
formation efficiency of the fragments observed in the low mass range of TOF-SIMS spectra. However, because the high mass range of TOF-SIMS spectra can provide information for the monomeric unit sequence [17], molecular weight distribution [18], end group identification [19], and tertiary conformation $[20,21]$ of polymers in TOF-SIMS studies, high mass ranges obtained with different primary projectiles should be also compared.

In our previous work, Wells et al. reported the characterization of molecular weight distributions of poly(dimethylsiloxane) with polyatomic primary ions [22]. The results demonstrated that monatomic primary ions induced higher peak intensity with less fragmentation, especially in the determination of molecular weight distribution. This was attributed to the increased momentum transfer in a shallower surface region, creating interactions of the cluster primary ions with the top overlayer and more extensive bond breaking and fragmentation. This effect competed with the emission of intact oligomers necessary for molecular weight determination.

In order to understand the different bombardment mechanism between monatomic and polyatomic projectiles, in the present work we investigate the effect of various monatomic and polyatomic projectiles' bombardment on the fragmentation pattern of (sub)monolayer polymer samples in the high mass range of the TOF-SIMS spectrum. The polysiloxanes were deposited as a (sub)monolayer on silver substrates and the monatomic and polyatomic projectiles of $\mathrm{Cs}^{+}, \mathrm{Bi}_{1}{ }^{+}, \mathrm{Bi}_{3}$, and $\mathrm{C}_{60}{ }^{+}$were utilized for this study. In addition, the difference of fragmentation pattern of the (sub) monolayer polysiloxanes on silver substrates by Bi projectiles with different energy was also investigated.

\section{Experimental}

\section{Materials}

Poly(dimethylsiloxane)s (PDMS)s with $\mathrm{M}_{\mathrm{n}}$ of 2200 and 6140 were purchased from Polymer Source Inc. (Dorval, Quebec, Canada). PDMS-2200 and PDMS-6140 have polydispersity indices (PDI) of 1.09 and 1.07, respectively. Poly(methylphenylsiloxane) (PMPhS) with MW from 600 to 1000 was purchased from Gelest Inc. (Morrisville, PA, USA). Both PDMSs were terminated by sec-butyl and trimethylsiloxy groups; the end groups of PMPhS were not defined by the manufacturer. For the preparation of submonolayer films, the silver foil was purchased from SigmaAldrich Inc. (St. Louis, MO, USA) and the silver mirror used for manufacturing the Langmuir-Blodgett (LB) monolayer film was prepared following a reference method [21].

\section{Sample Preparation}

The PDMSs were dissolved in reagent grade toluene (Fisher Scientific, Suwanee, GA) with a concentration of $1 \mathrm{mg} / \mathrm{mL}$. For the solution casting method, $2 \mu \mathrm{L}$ of PDMS solution was deposited on each silver foil in sub-monolayer coverage. The silver foil was cleaned by ultrasonication in both hexane and toluene for $20 \mathrm{~min}$ each, and then etched in $10 \%$ nitric acid for $10 \mathrm{~min}$. The PMPhS in monolayer film was prepared by using LB technique. For the LB film preparation, PMPhS was dissolved in reagent grade pentane (Sigma Aldrich, Saint Louis, MO) with the concentration of $1 \mathrm{mg} / \mathrm{mL}$. In prior to the LB film preparation, the silver mirror was cleaned with $\mathrm{KOH} /$ isopropanol solution and washed with ultrapure water.

\section{Langmuir-Blodgett Film Transfer}

A computer-controlled KSV film balance was operated for LB film preparation. By using a cold trap, the temperature was decreased to $13^{\circ} \mathrm{C}$, at which the magnitude of the surface pressure was high enough for the PMPhS monolayer to be transferred onto the silver mirror. The surface pressure was measured with the accuracy of $0.1 \mathrm{mN} / \mathrm{m}$. The purified water from a Millipore Mega-Pure system, MP-6A, was used as subphase liquid. $30 \mu \mathrm{L}$ of PMPhS was spread onto the water subphase and then 3 min were required for the formation of a monolayer on the subphase through evaporation of pentane. The PMPhS monolayer was compressed at a constant rate of $5 \mathrm{~cm}^{2} /$ min up to the surface pressure of $2.0 \mathrm{mN} / \mathrm{m}$ before its collapse. At that surface pressure, then $\mathrm{PMPhS}$ monolayer was transferred onto the silver mirror with a dipping speed of $2 \mathrm{~mm} / \mathrm{min}$ upward.

\section{TOF-SIMS}

The TOF-SIMS used in this study was an ION TOF Model 5.100 (ION TOF Gmbh, Muenster, Germany) equipped with a $\mathrm{Bi}_{n}{ }^{+}$liquid metal ion source, and a dual source column containing $\mathrm{Cs}^{+}$and $\mathrm{C}_{60}{ }^{+}$sources. For all primary ions, total ion flux dosage during data acquisition was between $1.5 \times$ $10^{12}$ and $5.0 \times 10^{12}$ ions $/ \mathrm{cm}^{2}$, which is the accepted value within the static limit. The analyzer is of the reflection type; reflector voltage of $19 \mathrm{eV}$ and post-acceleration voltage of $10 \mathrm{keV}$ were applied for all spectra acquisitions. The pressure of the main chamber was kept between $5 \times 10^{-8}$ and $5 \times 10^{-9}$ mbar. ION SPEC software from ION TOF Gmbh (Muenster, Germany) was used for the data analysis.

\section{Results and Discussion}

The low mass range of the TOF-SIMS spectrum for PDMS deposited on silver substrate has been reported previously [19]. In our work, the dominant peaks corresponding to $\mathrm{Si}^{+}$, $\mathrm{CH}_{3} \mathrm{Si}^{+}, \mathrm{C}_{3} \mathrm{H}_{9} \mathrm{Si}^{+}$, and $\mathrm{C}_{5} \mathrm{H}_{15} \mathrm{OSi}_{2}{ }^{+}$were also observed at 28 , 43,73 , and $147 \mathrm{~m} / z$, respectively, and the spectra of PDMS2200 and PDMS-6140 were similar in this range. However, the $\mathrm{C}_{60}{ }^{+}$ion source yielded higher intensities of PDMS low mass fragment peaks compared to other sources and it was discussed in our previous work [22].

Figure 1 shows the TOF-SIMS spectrum with the mass range between 400 and $1000 \mathrm{Da}$ for PDMS-2200 under 

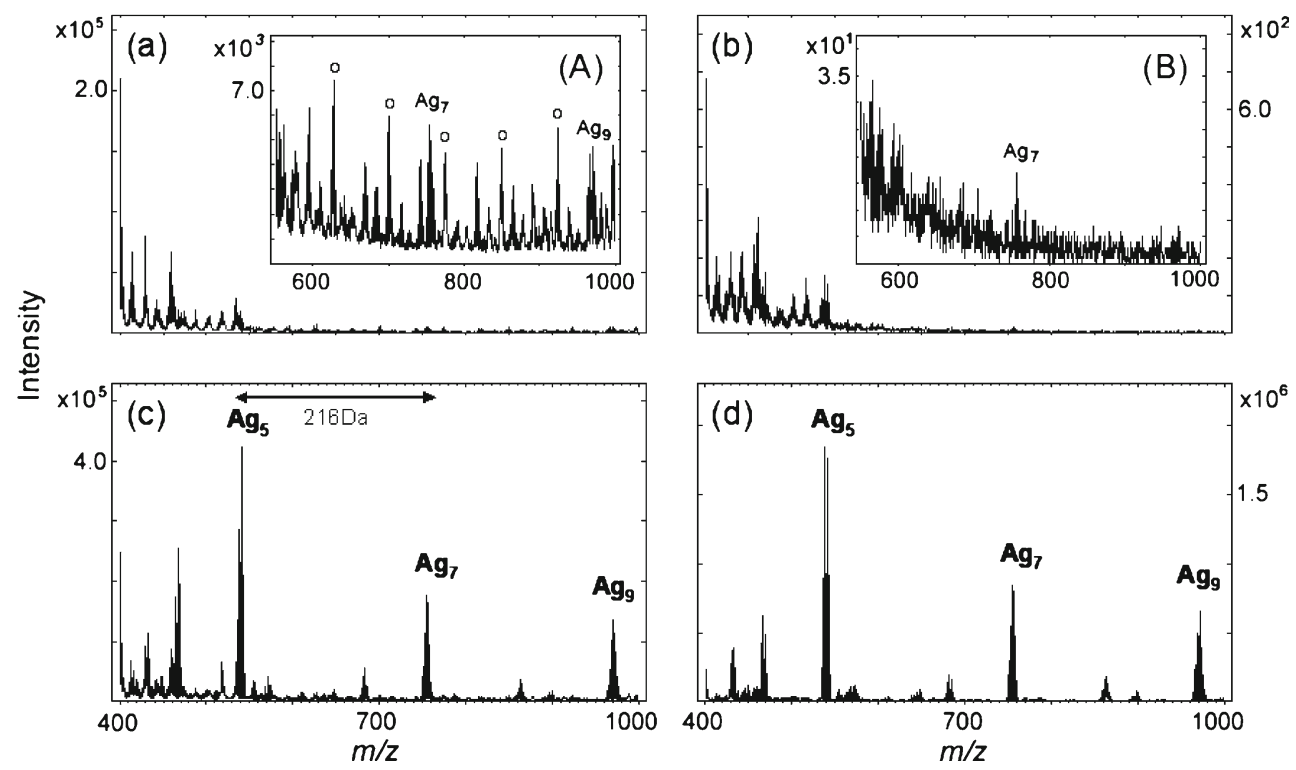

Figure 1. The mass range between 400 and $1000 \mathrm{Da}$ for PDMS-2200 under bombardment of $\mathrm{Cs}^{+}(10 \mathrm{keV})(\mathrm{a}), \mathrm{C}_{60}{ }^{+}(10 \mathrm{keV})$ (b), $\mathrm{Bi}_{1}{ }^{+}(25 \mathrm{keV})(\mathbf{c})$, and $\mathrm{Bi}_{3}{ }^{+}(25 \mathrm{keV})$ (d). The inserted spectra in (a) and (b) are the detailed PDMS-2200 spectra between 550 and $1000 \mathrm{Da}$ by $\mathrm{Cs}^{+}(10 \mathrm{keV})$ (a) and $\mathrm{C}_{60}{ }^{+}(10 \mathrm{keV})$ (b)

bombardment by $10 \mathrm{keV} \mathrm{Cs}^{+}$and $\mathrm{C}_{60}{ }^{+}$, and $25 \mathrm{keV} \mathrm{Bi}_{1}{ }^{+}$and $\mathrm{Bi}_{3}{ }^{+}$. Compared with $\mathrm{Cs}^{+}$and $\mathrm{C}_{60}{ }^{+}$, intense fragments corresponding to silver cluster ions with odd composition number were observed by $\mathrm{Bi}_{1}{ }^{+}$and $\mathrm{Bi}_{3}{ }^{+}$. The ejection of these silver cluster ions detected under the $\mathrm{Bi}_{1}{ }^{+}$and $\mathrm{Bi}_{3}{ }^{+}$ bombardment can be explained by the difference of atomic mass between the bismuth projectile and the substrate. The bismuth atom cannot be easily deflected by the silver atoms in the solid substrate due to its significantly heavier mass than that of the silver. Thus, the bismuth atoms propagate downward passing through the polymer overlayer and then transfer their energy to a considerably deep area for the silver atoms to be emitted into vacuum. Consequently, the ejection of the silver cluster ions from the substrate under the polymer overlayer in the (sub)monolayer was detected in TOF-SIMS spectra under all conditions of bismuth ion bombardment. Figure 1a and $\mathrm{b}$ show the detailed PDMS2200 spectra between 550 and $1000 \mathrm{Da}$ by $\mathrm{Cs}^{+}$and $\mathrm{C}_{60}{ }^{+}$. With the $\mathrm{Cs}^{+}$projectile, the fragments corresponding to cyclic oligomers cationized by silver (o) as well as silver
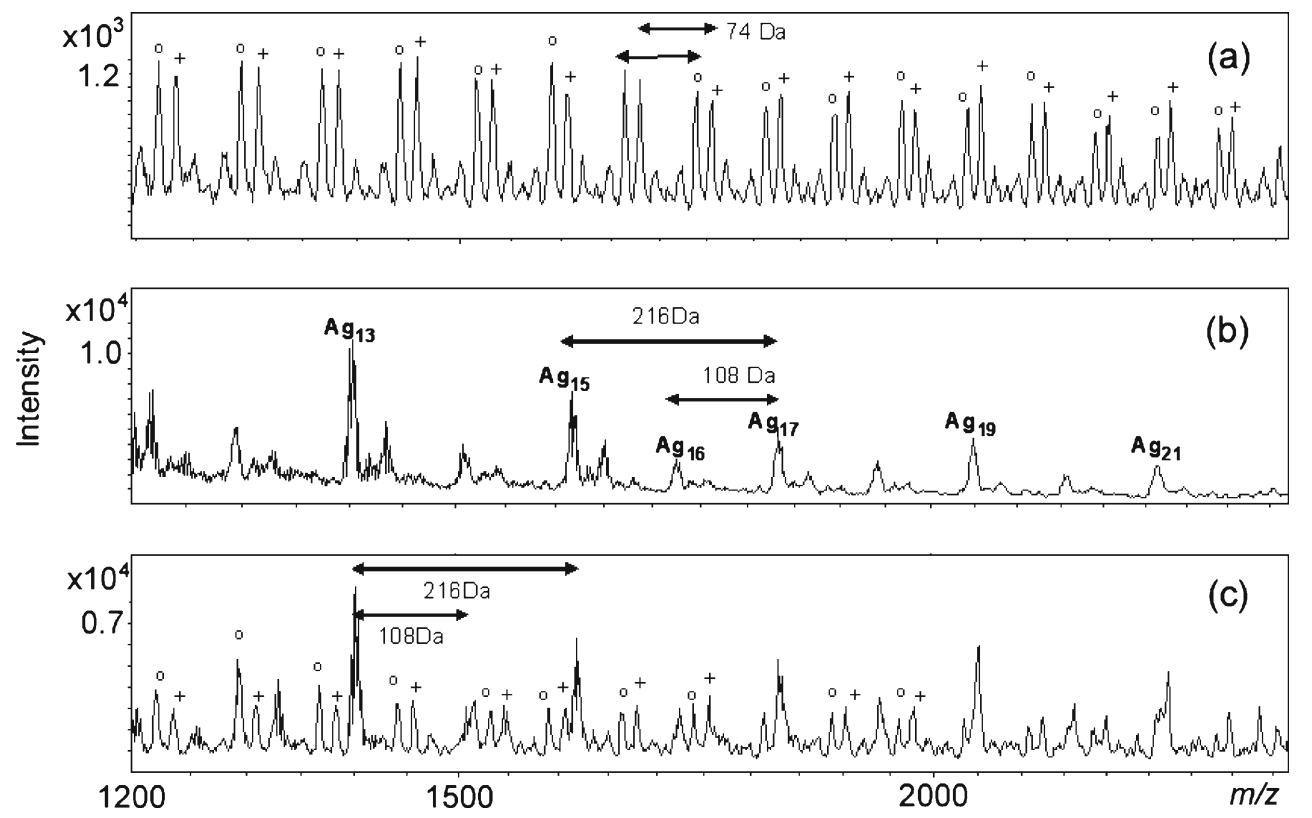

Figure 2. The mass range between 1200 and $2500 \mathrm{Da}$ for PDMS-6140 under $\mathrm{Cs}^{+}(10 \mathrm{keV})$ (a), $\mathrm{Bi}_{3}{ }^{+}$and $\mathrm{Bi}_{3}{ }^{++}(25 \mathrm{keV})$ (b), and $\mathrm{Bi}_{1}{ }^{+}(25 \mathrm{keV})$ (c) impact 


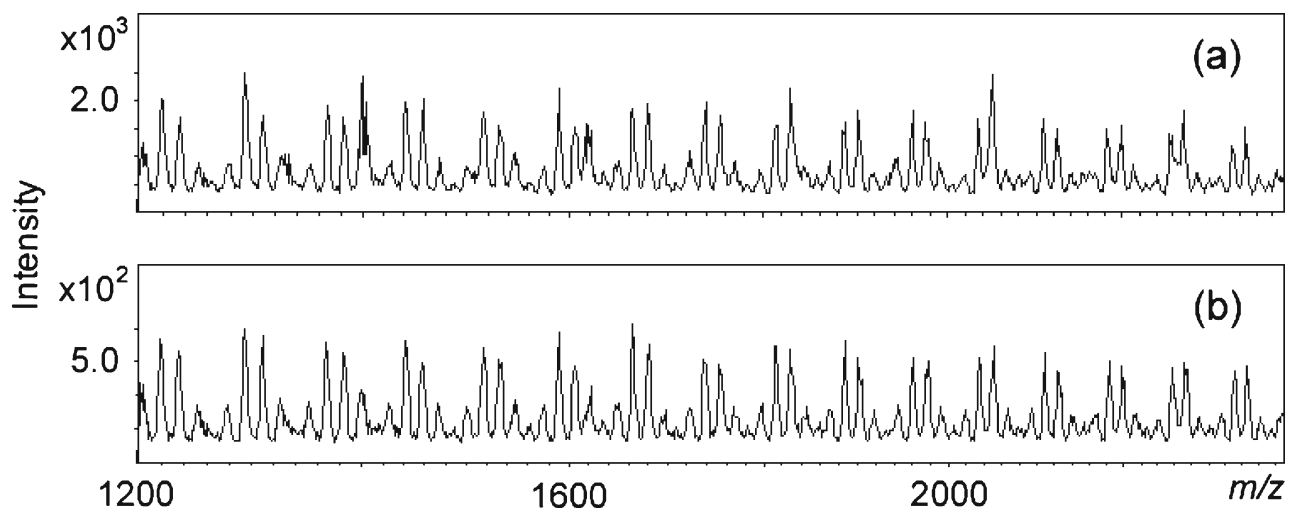

Figure 3. The mass range between 1200 and $2500 \mathrm{Da}$ for PDMS-6140 under bombardment by $\mathrm{Bi}_{1}{ }^{+}$(a) and $\mathrm{Bi}_{3}{ }^{+}$(b) $(15 \mathrm{keV})$

clusters were observed but only the $\mathrm{Ag}_{7}$ cluster ion with weak intensity was detected by $\mathrm{C}_{60}{ }^{+}$impact. Under both of $\mathrm{Cs}^{+}$and $\mathrm{C}_{60}{ }^{+}$impact, however, the intense silver cluster ions were not detected in the spectrum. In the case of a $\mathrm{Cs}^{+}$ projectile, penetration into the substrate occurs but desorption of only the intact and fragmented polymer overlayer occurs without extensive desorption of the silver ions from the substrate. This might be because the $\mathrm{Cs}^{+}$projectile is not sufficiently large and energetic to eject the silver ions. This result is consistent with that from the molecular dynamics simulation of the $\mathrm{Ga}^{+}$bombardment reported previously [11, 12]. In addition, each carbon atom on $\mathrm{C}_{60}{ }^{+}$impact cannot be embedded into the silver substrate and interacts strongly with the polymer overlayer because the carbon atoms are easily deflected by silver atoms. As a result, they deposit most of their energy in a shallower and broader volume of the sample so that the most particles are ejected from polymer overlayer.

For the higher molecular weight PDMS-6140, silver cluster ions were also observed in the particular high mass range of the TOF-SIMS spectrum under $\mathrm{Bi}_{1}{ }^{+}$and $\mathrm{Bi}_{3}{ }^{+}$ bombardment. Figure 2 shows the results from the mass

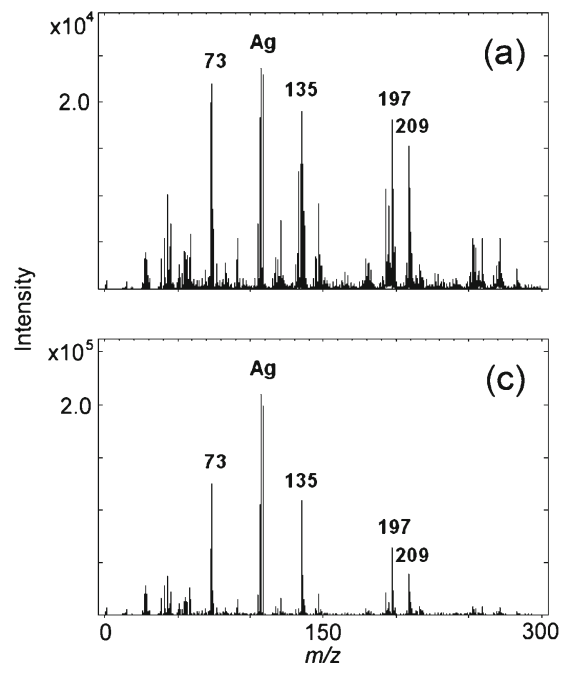

range between 1200 and $2500 \mathrm{Da}$ for PDMS-6140 from primary ions of $10 \mathrm{keV} \mathrm{Cs}^{+}, 25 \mathrm{keV} \mathrm{Bi}_{1}{ }^{+}$, and $\mathrm{Bi}_{3}{ }^{+}$. In the spectrum from $\mathrm{Cs}^{+}$bombardment, two intense clusters are observed: a pattern of fragments corresponding to cyclic oligomers cationized by silver, $[\mathrm{Rn}+\mathrm{Ag}]^{+}$(o) and a series of the silver-cationized linear PDMS oligomers having the end groups of $-\mathrm{H}$ and $-\mathrm{CH}_{3},[\mathrm{nR}+16+\mathrm{Ag}]^{+}(+)$. Rn and $\mathrm{nR}$ refer to a cyclic and a linear fragment containing an integral number (n) of repeating units, respectively. These two ion clusters were also observed under $\mathrm{Ar}^{+}$bombardment by Dong et al. [19], who attributed those peaks to the fragments produced by ion-beam bombardment, not to oligomers originally present in the sample. Under $\mathrm{Bi}_{1}{ }^{+}$impact shown in Figure 2, not only were the ion clusters of $[\mathrm{Rn}+\mathrm{Ag}]^{+}$and $[\mathrm{nR}+16+\mathrm{Ag}]^{+}$still observed, but also silver cluster ions with odd number composition from $\mathrm{Ag}_{13}$ to $\mathrm{Ag}_{21}$ were detected. However, in case of the $\mathrm{Bi}_{3}{ }^{+}$bombardment, only silver cluster ions were observed. The larger atomic mass of the $\mathrm{Bi}$ projectile compared with that of $\mathrm{Cs}^{+}$induced emission of the large silver cluster ions from the substrate as well as two series of silver cationized fragments from the polymer overlayer. Upon impact of polyatomic $\mathrm{Bi}$, however, the

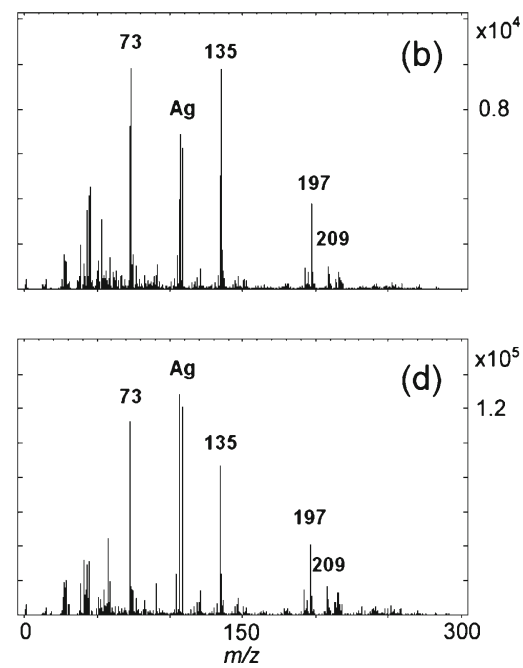

Figure 4. The mass range below $300 \mathrm{Da}$ for $\mathrm{PMPhS}$ with $\mathrm{MW}$ from 600 and $1000 \mathrm{Da}$ under $\mathrm{Cs}^{+}(10 \mathrm{keV})(\mathbf{a}), \mathrm{C}_{60}{ }^{+}(10 \mathrm{keV})(\mathbf{b})$, $\mathrm{Bi}_{1}^{+}(25 \mathrm{keV})$ (c), and $\mathrm{Bi}_{3}^{+}(25 \mathrm{keV})$ (d) impact 

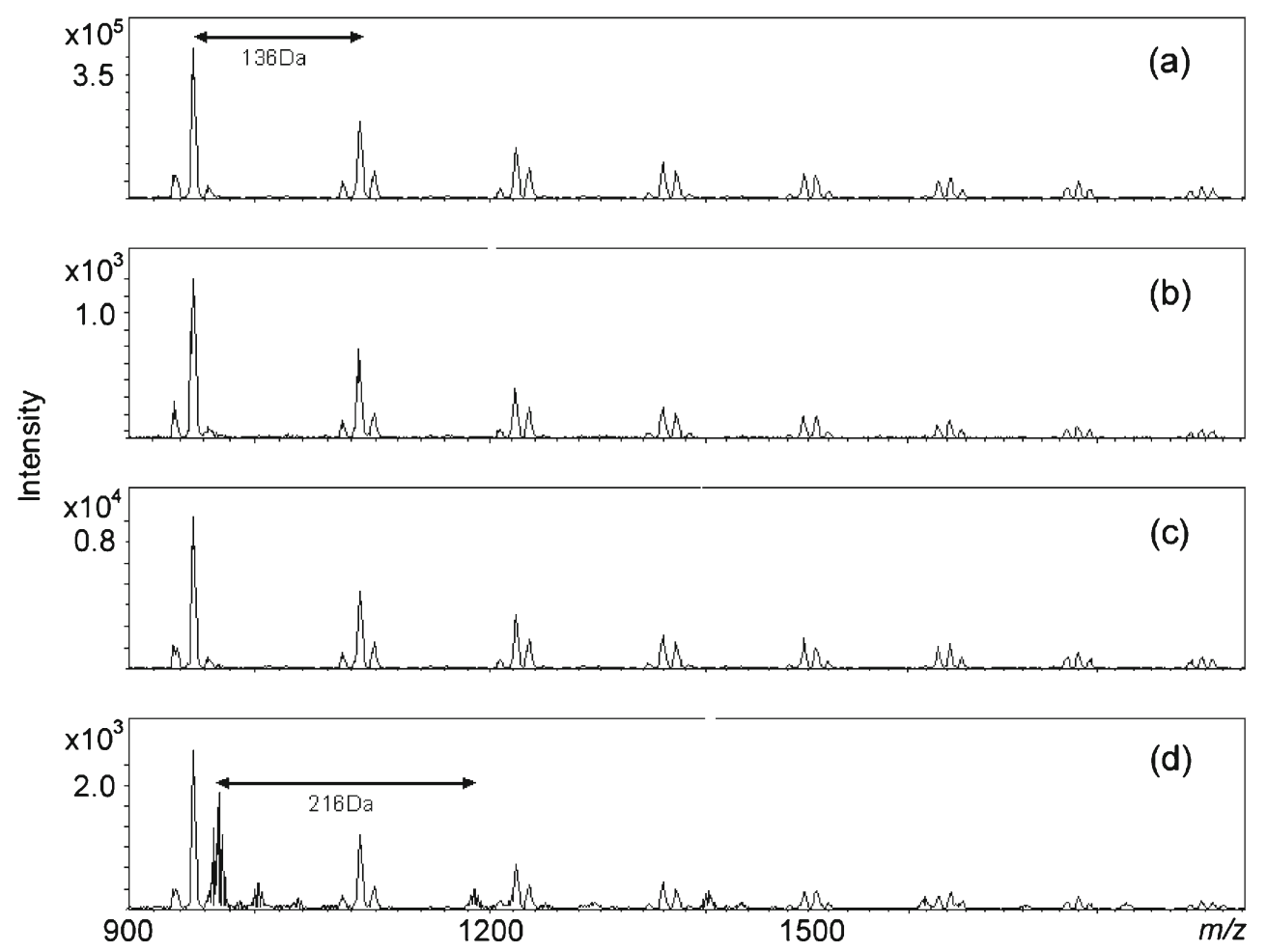

Figure 5. The mass range between 900 and $2000 \mathrm{Da}$ for PMPhS with MW from 600 and $1000 \mathrm{Da}$ under $\mathrm{Cs}^{+}(10 \mathrm{keV})(\mathrm{a}), \mathrm{C}_{60}{ }^{+}$ (10 keV) (b), $\mathrm{Bi}_{1}{ }^{+}(25 \mathrm{keV})$ (c), and $\mathrm{Bi}_{3}{ }^{+}(25 \mathrm{keV})$ (d) impact

silver-cationized fragments from the polymer overlayer were not detected. This indicates that the main portion of the polyatomic bismuth projectile's momentum was used to emit the silver cluster ions or small fragments, and not to break down the intact polymer overlayer into large size fragments to be emitted into vacuum. This may result from the polyatomic Bi projectile having a deeper sampling depth than monatomic $\mathrm{Bi}$ ions, due to the split trajectories of the component atoms [22]. Because triatomic Bi bombardment ejects only silver clusters in this mass range, we can say that it could not provide information related to the high mass ions fragmented from the polymer overlayer on the silver substrate.

Beyond the factors such as ion size and, for primary ions, the trajectories of component atoms that determine the depth of penetration, the effect of Bi primary ion energy on the fragmentation of polymer in the high mass range of the TOF-SIMS spectrum was also investigated in this study. Figure 3 shows the TOF-SIMS spectrum of the medium mass range between 1200 and 2500 Da for PDMS-6140, bombarded by $15 \mathrm{keV} \mathrm{Bi}_{1}{ }^{+}$and $\mathrm{Bi}_{3}{ }^{+}$projectiles. While the pattern of ion clusters under the impact of $15 \mathrm{keV} \mathrm{Bi}_{1}{ }^{+}$was similar to that with $25 \mathrm{keV} \mathrm{Bi}_{1}{ }^{+}$, two series of ion clusters corresponding to $[\mathrm{Rn}+\mathrm{Ag}]^{+}$and $[\mathrm{nR}+16+\mathrm{Ag}]^{+}$as well as the silver cluster ions were much less intense than observed with $15 \mathrm{keV} \mathrm{Bi}_{3}{ }^{+}$. Because $25 \mathrm{keV} \mathrm{Bi}_{3}{ }^{+}$induces only silver cluster ions in this mass range, we can conclude, as a result, that depending on the type of projectile, the kinetic energy can be one of the parameters that influences the fragmentation pattern of the polymer.

In the present study, the fragmentation pattern in the TOF-SIMS spectrum of the LB film of poly(methylphenylsiloxane) (PMPhS) is also reported. Figure 4 shows the low mass range below $300 \mathrm{Da}$ for PMPhS with MW from 600 to $1000 \mathrm{Da}$ under each impact of $10 \mathrm{keV} \mathrm{Cs}^{+}$and $\mathrm{C}_{60}{ }^{+}$, and $25 \mathrm{keV} \mathrm{Bi}_{1}{ }^{+}$and $\mathrm{Bi}_{3}{ }^{+}$. With all projectiles, the intense peaks are observed at $73,135,197$, and $209 \mathrm{Da}$, which correspond to $\left(\mathrm{CH}_{3}\right)_{3} \mathrm{Si}^{+},\left[\left(\mathrm{CH}_{3}\right)_{2}(\mathrm{Ph}) \mathrm{Si}\right]^{+},\left[\left(\mathrm{CH}_{3}\right)(\mathrm{Ph})_{2} \mathrm{Si}\right]^{+}$, and $\left[\left(\mathrm{CH}_{3}\right)\right.$ $\left.{ }_{3} \mathrm{SiOSi}(\mathrm{Ph})\left(\mathrm{CH}_{3}\right)\right]^{+}$, respectively. These peaks were observed for all projectile analyses used in this study and among them

Table 1. Summary of peak intensity ratios of summed high mass peak intensities of PMPhS and PDMS-2200 referenced to the total ion intensities, respectively

\begin{tabular}{|c|c|c|c|c|}
\hline & \multicolumn{4}{|l|}{ Bombarding species } \\
\hline $\begin{array}{l}\text { PMPhS } \\
\text { PDMS-2200 }\end{array}$ & $\begin{array}{l}(1.63 \pm 0.84) \times 10^{-2} \\
(1.96 \pm 0.49) \times 10^{-2}\end{array}$ & $\begin{array}{l}(4.34 \pm 3.63) \times 10^{-3} \\
(1.21 \pm 0.25) \times 10^{-2}\end{array}$ & $\begin{array}{l}(1.40 \pm 0.53) \times 10^{-2} \\
(3.05 \pm 0.54) \times 10^{-2}\end{array}$ & $\begin{array}{l}(1.17 \pm 0.03) \times 10^{-4} \\
(1.35 \pm 0.41) \times 10^{-5}\end{array}$ \\
\hline
\end{tabular}


Bi projectiles yielded higher peak intensities of the low mass fragments. When we compared the peak intensities of characteristic peaks of PMPhS $(73 \mathrm{Da}$ and $135 \mathrm{Da})$ referenced to the intensities of silver substrate peaks $(107 \mathrm{Da}$ and $109 \mathrm{Da})$, however, Figure 4 reveals that $\mathrm{C}_{60}{ }^{+}$ bombardment induces their higher intensity ratio. This result is in good agreement with our previous work, in which it was discussed that the higher intensity ratios of the low mass PDMS fragments referenced to silver peaks were yielded by $\mathrm{C}_{60}{ }^{+}$impact [22].

Figure 5 shows the high mass range between 900 and $2000 \mathrm{Da}$ for PMPhS by $10 \mathrm{keV} \mathrm{Cs}^{+}$and $\mathrm{C}_{60}{ }^{+}$, and $25 \mathrm{keV}$ $\mathrm{Bi}_{1}{ }^{+}$and $\mathrm{Bi}_{3}{ }^{+}$. The pattern of ion clusters corresponding to the silver cationized intact oligomer was observed for all projectiles. Each of those ion clusters is separated by an interval equal to a monomer unit mass of $136 \mathrm{Da}$. Under the impact of $\mathrm{Bi}_{3}{ }^{+}$, however, the series of the silver cluster ions as well as the intact PMPhS oligomer cationized by silver was observed. Such ejection of silver cluster ions might be due to a deeper sampling depth resulting from the split trajectories of component atoms under the polyatomic impact. This indicates that the momentum transfer of the Bi polyatomic projectile leads to the ejection of the silver cluster ions even with high mass.

In the PMPhS and PDMS spectra acquired with monatomic and polyatomic projectiles, the differences of intensities of intact oligomer cationized by silver were observed. For the reliable comparison, Table 1 shows a plot of the ratios of summed peak intensities of intact oligomer cationized by silver for PDMS-2200 and $\mathrm{PMPhS}$ referenced to total ion intensities with different primary ions, respectively. For both PMPhS and PDMS, $\mathrm{C}_{60}{ }^{+}$analysis yielded significantly lower intensities of silver-cationized intact oligomer than the other analysis. Because $\mathrm{C}_{60}{ }^{+}$interacts with the polymer overlayer in a shallower volume, it could be giving rise to more small fragments and not lifting the intact polymer chains off the surface. This is evidence of a fundamental difference in the interaction of the $\mathrm{C}_{60}{ }^{+}$with the surface. The difference between monatomic and polyatomic bismuth projectiles was also informative; $\mathrm{Bi}_{1}{ }^{+}$analysis induced higher intensity ratios compared to $\mathrm{Bi}_{3}{ }^{+}$. This might be also due to their different sampling depths previously discussed. From this quantitative difference of the intensity ratios, we can say that monatomic primary ion sources yield higher peak intensities of intact polysiloxane oligomers observed in the high mass range of TOFSIMS spectra. Thus, it can be verified that monatomic ion analysis is more efficient for the study of high mass range polymers on metal substrates in SIMS, supporting our previous study [22].

\section{Conclusion}

We have reported the differences of the fragmentation patterns in the high mass range of TOF-SIMS spectra for the (sub)monolayer film of polysiloxanes under bombardment of $\mathrm{Cs}^{+}, \mathrm{C}_{60}{ }^{+}(10 \mathrm{keV}), \mathrm{Bi}_{1}{ }^{+}$, and $\mathrm{Bi}_{3}{ }^{+}(15$ and $25 \mathrm{keV})$. Twentyfive $\mathrm{keV}$ Bi projectiles emitted the silver cluster ions from the substrate under the polymer overlayer due to its heavy atomic mass and energetic impact velocity. The emission of silver cluster ions depended on the change of energy and the component atom number of $\mathrm{Bi}$ projectile. In the case of $\mathrm{Bi}_{3}{ }^{+}$, the silver cluster ions with much larger mass were detected, and $15 \mathrm{keV} \mathrm{Bi}_{3}{ }^{+}$showed a different fragmentation pattern from that of $25 \mathrm{keV} \mathrm{Bi}_{3}{ }^{+}$. Under impact of $\mathrm{Cs}^{+}$and $\mathrm{C}_{60}{ }^{+}$, however, silver cluster ions were not detected. Also, bombardment by $\mathrm{C}_{60}{ }^{+}$did not lift off the intact oligomer present in the PDMS-6140 polymer overlayer. From these results, the different patterns of fragmentation under the various projectile bombardments could be explained by their possible impact mechanisms. Additionally, monatomic projectiles yielded higher intensities of intact oligomer cationized peaks observed in the high mass range for PMPhS and PDMS. Therefore, we can confirm that there is no benefit to the use of polyatomic projectiles for the study of the characteristic fragmentation observed in high mass range of TOF-SIMS spectra of polymers in (sub)monolayers.

\section{Acknowledgments}

The authors acknowledge support in part for this research by the National Science Foundation Chemistry Division, grant CHE0616916. The authors gratefully acknowledge the generous support of Professor Thomas Beebe at the University of Delaware for HKM.

\section{References}

1. Castner, D.G.: Surface science: View from the edge. Nature 422, 129-130 (2003)

2. Winograd, N.: The magic of cluster SIMS. Anal. Chem. 77, 142A-149A (2005)

3. Wucher, A.: Molecular secondary ion formation under cluster bombardment: A fundamental review. Appl. Surf. Sci. 252, 6482-6489 (2006)

4. Kötter, F., Benninghoven, A.: Secondary ion emission from polymer surfaces under $\mathrm{Ar}^{+}, \mathrm{Xe}^{+}$and $\mathrm{SF}_{5}^{+}$Ion bombardment. Appl. Surf. Sci. 133, 47-57 (1998)

5. Weibel, D., Wong, S., Lockyer, N., Blenkinsopp, P., Hill, R., Vickerman, J. C.: $\mathrm{A} \mathrm{C}_{60}$ primary ion beam system for the time of flight secondary ion mass spectrometry: Its development and secondary ion yield characteristics. Anal. Chem. 75, 1754 (2003)

6. Weibel, D.E., Lockyer, N., Vickerman, J.C.: $\mathrm{C}_{60}$ cluster ion bombardment of organic surfaces. Appl. Surf. Sci. 231/232, 146-152 (2004)

7. Davies, N., Weibel, D.E., Blenkinsopp, P., Lockyer, N., Hill, R., Vickerman, J.C.: Development and experimental application of a gold liquid metal ion source. Appl. Surf. Sci. 203/204, 223-227 (2003)

8. Kersting, R., Hagenhoff, B., Kollmer, F., Möllers, R., Niehuis, E.: Influence of primary ion bombardment conditions on the emission of molecular secondary ions. Appl. Surf. Sci. 231/232, 261-264 (2004)

9. Koller, F.: Cluster primary ion bombardment of organic materials. Appli. Surf. Sci 231/232, 153-158 (2004)

10. Postawa, Z.: Sputtering simulations of organic overlayers on metal substrates by monoatomic and clusters projectiles. Appl. Surf. Sci. 231/ 232, 22-28 (2004)

11. Postawa, Z., Czerwinski, B., Winograd, N., Garrison, B.J.: Microscopic insights into the sputtering of thin organic films on $\operatorname{Ag}\{111\}$ induced by C60 and Ga Bombardment. J. Phys. Chem. B 109, 11973-11979 (2005)

12. Czerwiński, B., Samson, R., Garrison, B.J., Winograd, N., Postawa, Z.: Desorption of organic overlayers by $\mathrm{Ga}$ and $\mathrm{C}_{60}$ bombardment. Vacuum 81, 167-173 (2006) 
13. Russo, M.F., Garrison, B.J.: Mesoscale energy desorption footprint model for Kiloelectronvolt cluster bombardment of solids. Anal. Chem. 78, 7206-7210 (2006)

14. Russo, M.F., Ryan, K.E., Czerwinski, B., Smiley, E.J., Postawa, Z., Garrison, B.J.: Combined simulations and analytical model for predicting trends in cluster bombardment. Appl. Surf. Sci. 255, 897-900 (2008)

15. Staple, D., Brox, O., Benninghoven, A.: Secondary-ion emission from Arachidic acid LB-layers under $\mathrm{Ar}^{+}, \mathrm{Xe}^{+}, \mathrm{Ga}^{+}$, and $\mathrm{SF}_{5}^{-}$primary ion bombardment. Appl. Surf. Sci. 140, 156-167 (1998)

16. Stapel, D., Thiemann, M., Benninghoven, A.: Secondary ion emission from polymethacrylate LB-layers under $0.5-11 \mathrm{keV}$ atomic and molecular primary ion bombardment. Appl. Surf. Sci. 158, 362-374 (2000)

17. Dong, X., Gusev, A., Hercules, D.M.: Characterization of polysiloxanes with different functional groups by time-of-flight secondary ion mass spectrometry. J. Am. Soc. Mass Spectrom. 9, 292-298 (1998)

18. Elman, J.F., Lee, D.H.-T., Koberstein, J.T.: Time-of-flight secondary ion mass spectrometric measurements of molecular weight distributions for functionally terminated oligomers and transferred Langmuir-Blodgett-Kuhn monolayers. Langmuir 11, 2761-2767 (1995)

19. Dong, X., Proctor, A., Hercules, D.M.: Characterization of poly (dimethylsiloxane)s by time-of-flight secondary ion mass spectrometry. Macromolecules 30, 63-70 (1997)

20. Rey-Santos, R., Piwowar, A., Alvarado, L.Z., Gardella Jr., J.A.: SIMS studies of polymeric tertiary structures in monolayers: Polysiloxane helical coild structures. Appl. Surf. Sci. 252, 6605-6608 (2006)

21. Piwowar, A.M., Gardella Jr., J.A.: Time-of-flight secondary ion mass spectrometric analysis of polymer tertiary structure in langmuir monolayer films of poly(dimethylsiloxane). Anal. Chem. 79, 4126-4134 (2007)

22. Wells, D.D., Moon, H.K., Gardella Jr., J.A.: Time of flight secondary ion mass spectrometric determination of molecular weight distributions of low polydispersity poly(Dimethyl Siloxane) with polyatomic primary ions. J. Am. Soc. Mass Spectrom. 20, 1562-1566 (2009) 